\title{
Button batteries
}

\section{BATTERY INGESTION HAZARD MITIGATION}

doi:10.1136/injuryprev-2012-040590i.1

D Lee, J Midgett. US Consumer Product Safety Commission, USA

Background Recent research indicates an increase in button/coin battery ingestion, causing severe injuries or death, if not detected and removed within $2 \mathrm{~h}$ of lodging in the oesophagus. A multifaceted approach is needed to combat the hazard, including increasing consumer education and awareness of proper use and disposal of batteries; improving product standards for electronic enclosures, product packaging, and literature; and advancing technological improvements in battery design that could mitigate the hazard.

Objective To discuss conceptual technological improvements in button/coin battery designs to mitigate battery ingestion hazards.

Methods This paper considers various methods of reducing the hazard including: using nonconductive encapsulation or modifying the terminal surface area to reduce accessible electrode area; adding a protective device to limit current flow; or adding a collapsible mechanism to a battery's outer surface that will expand when the battery is removed from an enclosure so that it will be too big to be swallowed.

Results Practicalities of these conceptual changes and results of some battery ingestion simulations are discussed. Simulations show that near-complete encapsulation of one of the poles is necessary to prevent necrosis.

Significance Methods for battery ingestion prevention or disabling the current from an ingested battery could prevent or reduce severe injury and death. 